\title{
Conductance of a phenylene-vinylene molecular wire: Contact gap and tilt angle dependence
}

\author{
A. Bilić* \\ CSIRO Mathematics, Informatics and Statistics, Private Bag 33, Clayton South 3169 VIC, Australia \\ and Materials Theory and Simulation Laboratory, Institute of High Performance Computing, \\ 1 Fusionopolis Way, \#16-16 Connexis, Singapore 138632, Singapore \\ Ž. Crljen \\ R. Bošković Institute, P.O. Box 180, 10002 Zagreb, Croatia \\ B. Gumhalter \\ Institute of Physics, Bijenička 46, 10000 Zagreb, Croatia \\ J. D. Gale \\ Nanochemistry Research Institute, Department of Chemistry, Curtin University of Technology, \\ P.O. Box U1987, Perth, 6845 Western Australia, Australia \\ I. Rungger and S. Sanvito \\ School of Physics and CRANN, Trinity College, Dublin, Ireland \\ (Received 22 November 2009; revised manuscript received 4 February 2010; published 2 April 2010)
}

\begin{abstract}
Charge transport through a molecular junction comprising an oligomer of $p$-phenylene-vinylene between gold contacts has been investigated using density-functional theory and the nonequilibrium Green's function method. The influence of the contact gap geometry on the transport has been studied for elongated and contracted gaps, as well as various molecular conformations. The calculated current-voltage characteristics show an unusual increase in the low bias conductance with the contact separation. In contrast, for compressed junctions the conductance displays only a very weak dependence on both the separation and related molecular conformation. However, if the contraction of the gap between the electrodes is accommodated by tilting the molecule, the conductance will increase with the tilting angle, in line with experimental observations. It is demonstrated that the effect of tilting on transport can be interpreted in a similar way to the case of the stretching the junction with a molecule in an upright position. The lowest conductance was observed for the equilibrium gap geometry. With the dominant transport contribution arising from the $\pi$ system of the frontier junction orbitals, all the predicted increases in the conductance arise simply from the better band alignment between relevant frontier orbitals at the nonequilibrium geometries at the expense of weaker coupling with the contacts.
\end{abstract}

DOI: 10.1103/PhysRevB.81.155101

PACS number(s): $85.65 .+\mathrm{h}, 71.15 . \mathrm{Mb}$

\section{INTRODUCTION}

Over the past decade greater interest has arisen in molecular electronics, a rapidly growing field that is a prospective successor to metal-oxide-semiconductor based electronics (for a recent review see Ref. 1 and references therein). Electron transport through molecular junctions has been exploited to fabricate two- and three-terminal devices such as rectifiers, memory switches, and transistors. However, there remain many challenges to this promising technology, such as device stability, reproducibility, and control over the transport. Oligomers of $p$-phenylene-vinylene provide a promising platform for the fabrication of high conductivity molecular devices. The structural motif of oligo$p$-phenylene-vinylene (OPV) consists of coplanar phenyl moieties connected by vinylene links, which provide the conformational rigidity through a high rotational barrier and, at the same time, extend the conjugation of the system. Owing to these properties phenylene-vinylene polymers are frequently used in the synthesis of organic semiconductor devices. $^{2}$ Recently, thiol-capped OPV derivatives deposited between metal electrodes have been exploited for the fabri- cation of two- and three-terminal devices. ${ }^{3-6}$ In parallel to the experimental studies, significant progress has been made in the modeling of molecular junctions, although quantitative agreement between theory and experiments has remained elusive. . $^{7,8}$

The present computational work reports on the dependence of the conductance of a molecular junction comprising a thiol-capped OPV derivative with three-rings (OPV3) between gold contacts as a function of the contact gap separation. Both elongation and contraction of the junction away from the equilibrium spacing have been studied. In addition, the effects of the molecular tilting are investigated. For the sake of simplicity, extensive reconstructions of the gold contact surfaces ${ }^{9-11}$ are not considered here. We find a general trend of enhanced conductance as the geometry deviates from the upright orientation of the molecular wire in the optimal contact gap. This effect invariably arises because of the reduced coupling strength at the contacts, which results in the more favorable alignment of the relevant frontier orbitals with the Fermi level of the electrodes. For the compressed junctions with a distorted OPV3 wire little variation of conductance is found, owing to the resilience of the con- 
jugated frontier molecular orbitals. However, when OPV3 is tilted a similar I-V curve to that of the elongated junctions is predicted, again because of the better band line-up arising through the reduced bonding at the interface. The latter findings, while in agreement with recent experimental observations of Haiss et al., ${ }^{12,13}$ offer a different interpretation of their results for related wires.

\section{COMPUTATIONAL METHODOLOGY}

Geometry optimizations were conducted using densityfunctional theory (DFT) as implemented in the SIESTA program. ${ }^{14,15}$ In the SIESTA methodology a linear combination of atomic orbitals is used to expand the Kohn-Sham eigenstates. Core electrons and nuclei are replaced by normconserving pseudopotentials in a fully separable form. ${ }^{16}$ The modified scheme of Troullier and Martins ${ }^{17}$ was exploited to generate pseudopotentials for $\mathrm{Au}, \mathrm{C}, \mathrm{H}$, and $\mathrm{S}$, with relativistic corrections included for $\mathrm{Au}, \mathrm{C}$, and $\mathrm{S}$. For the exchange and correlation potential the functional of Perdew, Burke, and Ernzerhof (PBE), ${ }^{18}$ a form of generalized gradient approximation (GGA), was used for both pseudopotential generation and in the actual calculations. The one-electron Kohn-Sham eigenstates were expanded in a basis of strictly localized $^{19}$ numerical pseudoatomic orbitals. ${ }^{20}$ Basis functions were obtained by finding the eigenfunctions of the isolated atoms confined within a sphere. The range of the atomic orbitals was chosen so as to obtain an energy increase of $7 \mathrm{mRy}$ arising due to the spherical confinement. This results in orbital confinement radii of $2.38-3.07$ and $3.74 \AA$ for the molecule and gold orbitals, respectively. For transport calculations smaller energy shifts have been recommended, ${ }^{21,22}$ but because of the rather limited surface area of the supercell (see below), the tighter confinement of the orbitals is favorable, to minimize the overlap of periodic images of the molecule. Thus, even in the case of the most distorted geometry considered here, for which the minimal distance between two atoms placed in different periodic replica was $5.57 \AA$, the orbital overlap was still rather insignificant. A split-valence scheme was employed to generate a single- $\zeta$ basis set for the $6 s$ and $5 d$ states of $\mathrm{Au}$, and a double- $\zeta$ basis set for the $1 s$ state of $\mathrm{H}, 2 s$ and $2 p$ states of $\mathrm{C}$, $3 s$ and $3 p$ states of $\mathrm{S}$, with a single $-\zeta$ shell of polarization functions for all four elements. SIESTA uses an auxiliary real space mesh to evaluate terms based on charge density and an equivalent plane-wave cutoff of 350 Ry was used to determine the mesh spacing. All the atomic geometries were relaxed via conjugate gradient optimization until the forces were smaller $10 \mathrm{meV}^{-1}$. While SIESTA supports spinpolarized treatment of electrons, to reduce the computational cost only unpolarized calculations have been employed in the present work.

The optimized geometries from the SIESTA computations, as well as all the relevant computational parameters, were subsequently employed in electron transport calculations. The latter were conducted using the nonequilibrium Green's function (NEGF) Landauer approach ${ }^{23}$ as implemented in the SMEAGOL package, ${ }^{24-26}$ which is interfaced to SIESTA.
The two-terminal device was modeled using a supercell geometry of the $\mathrm{Au}(111)$ surface consisting of several atomic layers and a vacuum gap in which the OPV3 molecule was placed. The application of periodic boundary conditions along all the three Cartesian directions yields an infinite array of periodically repeated slabs separated by regions of vacuum. The gold slab was 10 atomic layers thick and the boundaries of the supercell were set up so that the single slab forms the two contacts, with $5 \mathrm{Au}$ atomic layers on each side. The system was then partitioned into two leads and a scattering region. The three layers of $\mathrm{Au}$ at the bottom and top of the cell (i.e., the inner six layers of the slab) were the leads and their geometries were fixed to the bulk positions. The two Au layers below and above the vacuum region (i.e., the outer two layers on each side of the slab) were taken as parts of the extended molecule, with which they form the scattering region (this is the part treated at the NEGF selfconsistent level). The geometry of the extended molecule was always optimized prior to the transport calculation. The thickness of vacuum, i.e., the separation of the electrodes varied from 19.57 to $24.57 \AA$. A single OPV3 molecule was inserted in the middle of the vacuum region, with $\mathrm{S}$ atoms approximately above the threefold hollow sites. However, because of the A-B-C stacking sequence of atomic layers on the gold (111) surface, the two faces of the slabs are not fully symmetric. Thus, if the $\mathrm{S}$ binding site on one of the contacts is fcc hollow, its counterpart on the opposite contact is hep hollow. Consequently, the coupling at the thiolate-gold interface will be somewhat stronger for the former site. ${ }^{27}$

A $(3 \times 3)$ superstructure was employed for the $\mathrm{Au}(111)$ surface resulting in nine gold atoms per layer. This represents a moderate 1/9 OPV3 monolayer coverage, which guarantees that the molecules in neighboring cells are sufficiently separated (i.e., noninteracting). ${ }^{27-34}$ For the geometry optimizations, as well as for the transport computations, the Brillouin zone integrations were performed on a $3 \times 3 k$-point Monkhorst-Pack mesh in the plane of the surface. For the calculation of the bulk electronic structure of the gold leads the reciprocal space was sampled on a $3 \times 3 \times 100$ grid, with the denser sampling in the direction of transport. Finally, for the optimizations of the gas phase OPV3 in a large supercell only the $\Gamma$ point was used. In the transport calculations the complex part of the integral leading to the charge density is computed by using 25 energy points on the complex semicircle, 20 points along the line parallel to the real axis and nine poles. The integral over real energies necessary at finite bias is evaluated over at least 500 points. ${ }^{24,25}$

The lattice parameter of gold was obtained from bulk computations of total energy versus the size of the unit cell. While this property is needed as an input parameter to the subsequent supercell computations, calculations of this type are also useful as they provide indication of the accuracy of the computational approach. The Brillouin zone integration was performed on a Monkhorst-Pack $8 \times 8 \times 8 k$-point mesh. The fit to an equation of state gives a lattice constant of $4.196 \AA$ and a bulk modulus of $141 \mathrm{GPa}$. These values are in an acceptable agreement with those from experiments, $4.08 \AA$ and $170 \mathrm{GPa}$, and in excellent agreement with those evaluated with the PW91 functional by using an almost complete plane-wave basis set, $4.2 \AA$ and $150 \mathrm{GPa} .{ }^{28}$ The band 
<smiles>Sc1ccc(/C=C/c2ccc(/C=C/c3ccc(S)cc3)cc2)cc1</smiles>

\section{trans, trans}<smiles>Sc1ccc(/C=C/c2ccc(/C=C/c3ccc(S)cc3)cc2)cc1</smiles>

trans,cis
FIG. 1. The two isomers of OPV3

structure of gold evaluated with the single $\zeta$ plus polarization function (SZP) basis set also shows remarkable similarity with that from the plane-wave approach. The results from these simple tests thus justify the use of the modest SZP basis set for $\mathrm{Au}$.

OPV3, without thiol ends better known as 1,4distyrylbenzene, can take two conformations illustrated in Fig. 1, trans, cis and trans, trans. Because of the limited surface area of the supercell and narrower profile of the former conformation, most calculations employed this geometry. However, for the equilibrium contact separation, we have verified that the differences between the conductance of the wire in the two conformations are negligible. Therefore the trans,trans conformation was not further considered in the present study.

\section{RESULTS AND DISCUSSION}

\section{A. Wire elongation}

The equilibrium separation for the junction was estimated from the length of the OPV 3 molecule in the gas phase and previously calculated distances between sulfur and gold atoms on the surface for phenylthiol. ${ }^{27}$ It is commonly assumed that thiols adsorb on gold dissociatively, i.e., by breaking the S-H bond, although both chemisorbed and physisorbed species can be observed at equilibrium. ${ }^{35}$ Since dissociation is the dominant mechanism, we have considered the gas phase OPV3 in the dehydrogenated biradical form. The optimized S-S distance was $19.63 \AA$ for the trans,cis conformation. Assuming a distance of $2.52 \AA$ between the $\mathrm{S}$ atom and the three $\mathrm{Au}$ atoms at the fcc hollow site of the lower surface and $2.56 \AA$ at the hcp hollow site of the opposite, upper face, the equilibrium contact distance is set to $23.07 \AA$ for the OPV3 in the upright orientation. A subsequent optimization of the extended molecule did not cause any notable change in the geometry. The calculated I-V characteristics is shown in the upper panel of Fig. 2. It is interesting to compare it with the one previously reported for

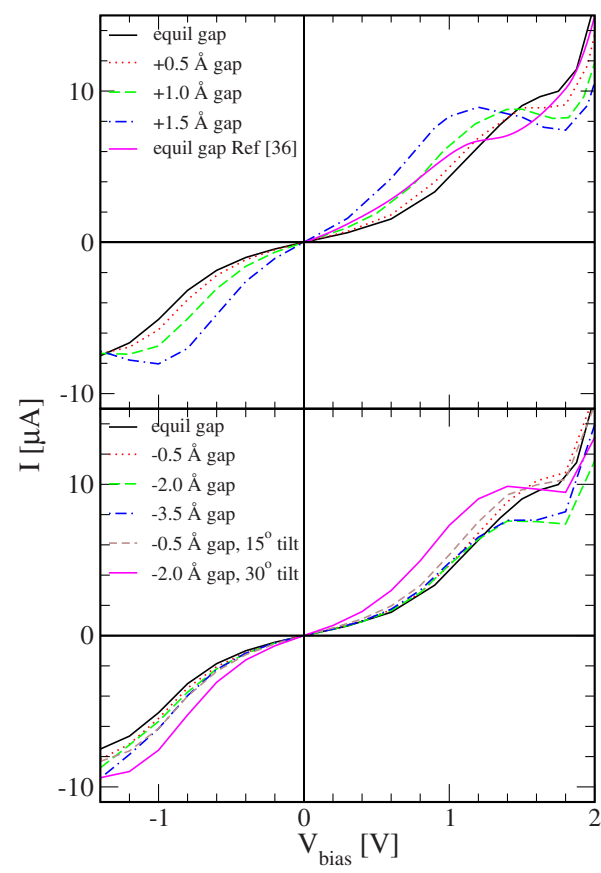

FIG. 2. (Color online) Upper panel: current vs bias voltage for the junctions stretched away from the equilibrium geometry. Lower panel: current vs bias voltage for the junctions contracted starting from the equilibrium geometry.

OPV $3,{ }^{36}$ also shown in the upper panel of Fig. 2. Despite the qualitative agreement, the discrepancies between the two are clear: the previously published curve predicts a higher conductance for low bias and it saturates earlier. For high biases the two curves are in rather good agreement. The calculations in Ref. 36 employed symmetric electrodes with molecule adsorbed symmetrically in hollow fcc positions on both electrodes. In the present calculations due to the adopted geometry of the unit cell the molecule is adsorbed in fcc hollow position at one electrode, and in the hcp hollow position at the opposite one. An asymmetric coupling to the electrodes usually results in the asymmetric potential profile along the length of the molecule, having as a consequence different alignment of molecular levels for positive and negative bias. The effect is normally seen as a slight rectification. The I-V curves in Fig. 2 of the calculations presented in this paper clearly demonstrate the slightly asymmetric curves with respect to reversed bias voltages, as opposed to the results presented in Ref. 36, where the curves were symmetric with respect to bias direction. In addition, a part of the difference in the I-V curves arises due the combination of different pseudopotentials, basis sets, and exchangecorrelation flavors (LDA in Ref. 36 vs GGA here), and in particular due to the different partition of the system into the two leads and extended molecule.

From the equilibrium separation, the contact distance was then gradually increased in steps of $0.5 \AA$ to a total of $1.5 \AA$ extra gap elongation. For each step the molecule was placed in the gap and optimized. Our calculations have confirmed that, for these moderate junction elongations, OPV3 favorably binds to both leads, rather than to a single side of the junction. 
TABLE I. Low bias conductance, $\sigma$, of the OPV3 wire for the considered junction geometries. $\Delta \mathrm{S}-\mathrm{S}$ is the difference in the separation between two $\mathrm{S}$ atoms relative to the distance of $19.63 \AA$ for an isolated biradical.

\begin{tabular}{lcc}
\hline $\begin{array}{l}\text { Gap separation } \\
(\AA)\end{array}$ & $\begin{array}{c}\Delta \mathrm{S}-\mathrm{S} \\
(\AA)\end{array}$ & $\begin{array}{c}\sigma \\
(\mu \mathrm{S})\end{array}$ \\
\hline+1.5 & +0.64 & 5.30 \\
+1.0 & +0.45 & 3.73 \\
+0.5 & +0.25 & 2.87 \\
Equilibrium & +0.03 & 2.40 \\
-0.5 & -0.43 & 2.56 \\
-1.0 & -1.20 & \\
-2.0 & -2.32 & 2.55 \\
-3.5 & -3.93 & 2.57 \\
$-0.5,15^{\circ}$ tilted & +0.05 & 2.88 \\
$-2.0,30^{\circ}$ tilted & +0.20 & 3.87 \\
\hline \hline
\end{tabular}

The calculated I-V characteristics for the stretched junctions are shown in the upper panel of Fig. 2. Most of the curves follow the familiar trend observed for the conjugated, thiol-capped, molecular wires between gold contacts; a slow increase in the current with the voltage for a low bias, followed by a plateau or a drop, which results in a negative differential resistance, and then a steeper increase. The I-V curves manifest a high degree of symmetry between positive and negative bias, which is expected given the rather symmetric geometry. It is interesting and counterintuitive that for low and moderate bias voltages the junctions with wider gap separation yield higher currents and, consequently, higher associated conductances at low bias, as Table I shows. This phenomenon has already been noticed for asymmetrically ${ }^{37-39}$ and symmetrically ${ }^{40}$ stretched junctions with the prototypical phenyldithiol molecular wire. It arose there because the peak in the transmission spectra associated with the highest occupied molecular orbital (HOMO) moved up in energy with increased contact separation, bringing it closer to the Fermi level. Thus, even though the coupling between the wire and the contacts gets weaker, it is more than compensated by the better level alignment of the relevant frontier orbitals.

\section{B. Wire compression}

Similarly to the case of elongated junctions, the geometries of the compressed junctions were computed by reducing the contact distance gradually in steps of $0.5 \AA$. The process of compression is illustrated in Fig. 3. In the first step, the molecule is only slightly bent away from that in equilibrium. However, with the following step an important conformational change takes place at the upper Au-S interface; from the hcp hollow adsorption site in the first panel, the upper $\mathrm{S}$ atom flips over the bridge site closer to the fcc binding site. This transformation, and subsequent steps, can be followed by looking at Fig. 4 of Ref. 27, which shows a two-dimensional potential-energy surface (PES) for phenylthiol on the $\mathrm{Au}(111)$ surface. When the gap is contracted
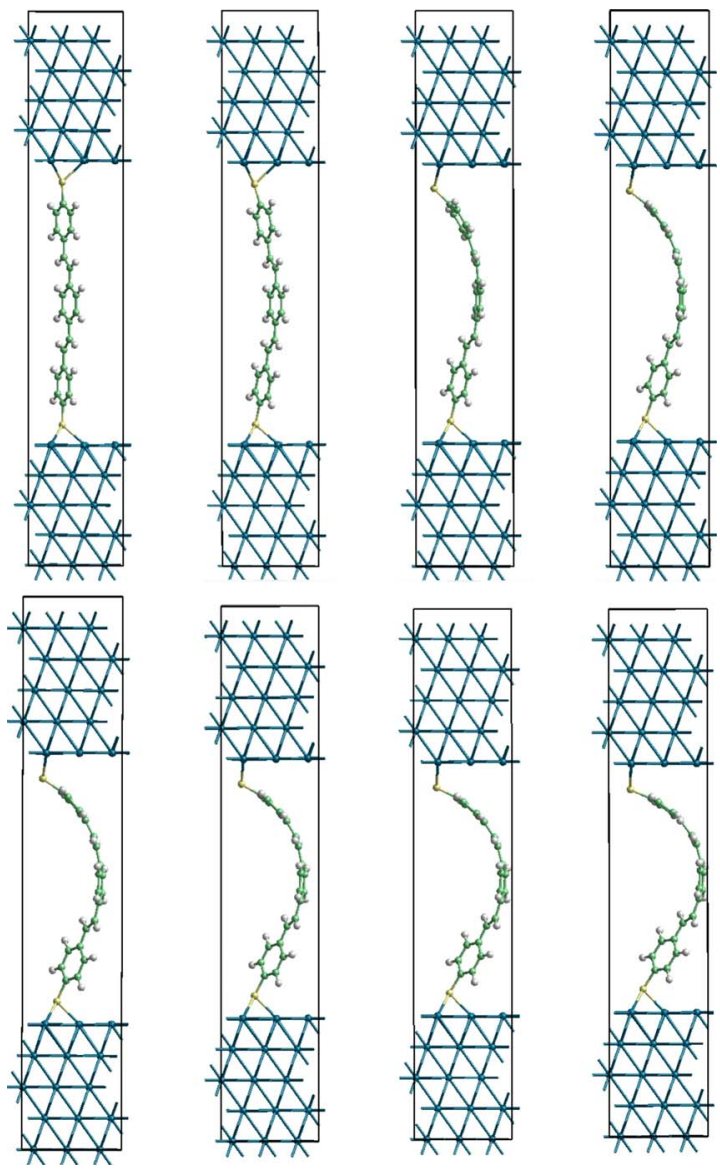

FIG. 3. (Color online) The effect of gradual compression, in steps of $0.5 \AA$, on the OPV3 wire, starting from the equilibrium geometry. Graphic produced with GDIS (Ref. 42) program.

by $1.0 \AA$ the binding site for the $\mathrm{S}$ atom changes from hcp (for phenyl group in upright orientation) to a site on the minimum energy path between fcc (phenyl group in upright orientation) and bridge (the plane of the phenyl group almost parallel with the surface). Then, as the gap separation is further reduced, the $\mathrm{S}$ atom is expected to shift slightly back, closer to the bridge site with its phenyl ring tilted closer to the surface. This is exactly what is observed in Fig. 3. Eventually, as the gap separation is reduced by $3.5 \AA$, the upper $\mathrm{S}$ atom is adsorbed vertically above the bridge site and its coordinating ring is tilted at a large angle away from the upright conformation.

The change in the nature of the S-Au bond at the upper surface over the series of compression steps is also reflected in the change of the S-S distance. As Table I shows, a gap compression of $0.5 \AA$ results in a smaller reduction in $0.43 \AA$ between the $\mathrm{S}$ ends. The difference of $0.07 \AA$ comes from the reduction in S-Au bond lengths, indicating the attractive interaction at the interfaces. However, during the next step, in which the gap is compressed by a total of $1.0 \AA$, the $\mathrm{S}$-S distance is reduced even more, by $1.20 \AA$. This shows that the molecule shrinks at the expense of the increased S-Au bond lengths, reflecting a more repulsive interaction at the interface. The interaction becomes increasingly more repulsive with every subsequent step, as the changes in the S-S distance relative to the gap contraction, 
a)

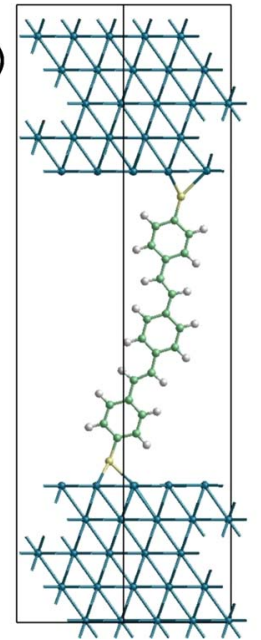

b)

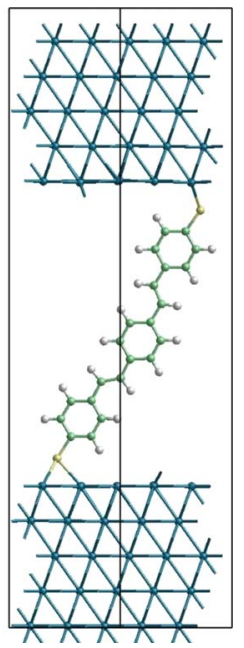

FIG. 4. (Color online) The orientation of the OPV3 wire in the (a) 0.5 and (b) $2.0 \AA$ contracted junction tilted at an angle of $15^{\circ}$ and $30^{\circ}$, respectively. Graphic produced with GDIS (Ref. 42) program.

shown in Table I, suggest. Interestingly, throughout this process the $\mathrm{S}$ atom on the lower face remains adsorbed on the fcc site and, in agreement with the previously computed $\mathrm{PES},{ }^{27}$ the associated phenyl ring, although tilted, remains closer to the upright orientation.

The calculated I-V characteristics for three compressed junctions are shown in the lower panel of Fig. 2. In contrast to those for the elongated junctions in the upper panel, these curves exhibit very little dispersion at low voltages. Hence, a low bias conductance of ca. $2.5 \mu \mathrm{S}$ is obtained for all three cases, as reported in Table I. The curve for the $0.5 \AA$ compressed junction deviates only marginally from that at equilibrium separation, which is expected given the similar geometry. However, even for the very distorted geometries, such as those at 2.0 and $3.5 \AA$ reduced separations, the associated characteristics exhibit substantial variance with the equilibrium curve only at voltages in excess of $1 \mathrm{~V}$.

It is clear that in a contracted junction the OPV3 wire does not need to adopt a bent conformation such as the one described above, but it can remain straight if it tilts away from the normal. In fact, in a typical experimental setup involving a rigid molecular wire, the reduced distance between the contacts usually results in tilting the molecular orientations away from the surface normal. ${ }^{12,13}$ Two tilted conformations have been considered, at $15^{\circ}$ and $30^{\circ}$ away from the normal, with contact gaps reduced by 0.5 and $2.0 \AA$ from the equilibrium, respectively, and the molecular plane perpendicular to the contact surfaces. Both situations are illustrated in Fig. 4. The lower S atom is again located at the fcc hollow site, while the upper S atom sits at the hop hollow and between the hcp hollow and bridge site for the 0.5 and $2.0 \AA$ compressed junctions, respectively. Hence, the adsorption sites and, presumably, the associated electronic couplings between the molecule and the electrodes, approximately match their counterpart bent conformations of Fig. 3 . However, the resulting currents, shown in the lower panel of Fig. 2, differ substantially. The tilted wires yield a better low bias conductance, as Table I shows. The characteristic of the

$30^{\circ}$ tilted OPV 3 wire, in particular, exhibits a higher conductance than any other compressed molecular junction considered in the present work.

\section{Discussion}

One of the interesting result of the present study is the notably enhanced conductance of OPV3 with the separation from the gold contacts. A similar effect has been predicted for other molecular wires attached to gold electrodes via thiol links. ${ }^{8,37-41}$ Unfortunately there is no experimental evidence of this behavior, e.g., recent measurements on related molecular wires have detected an opposite and expected trend, with the conductance increasing as the contact gap is reduced. ${ }^{12,13}$ However, it will be demonstrated below that the predicted characteristics of "rigid" molecular wires in stretched junctions might have already been manifested, although indirectly.

The study of the distorted wire in a compressed junction reveals more interesting details in the structural changes than in the electronic properties. The zero bias transmission spectra for the $0.5,2.0$, and $3.5 \AA$ contracted junctions are shown in the three bottom panels of Fig. 5. The main effect of the compression manifests in the contribution of the HOMO to the transmission, which shifts slightly closer to the Fermi level, resulting in slightly enhanced conductances, as Table I shows. Thus, as in the case of the extended junctions, the tradeoff between the better band alignment and the weaker contact coupling yields the higher conductance. Actually, as Table I shows, the minimum low bias conductance is predicted for the equilibrium geometry, where the bonding at the contacts is optimal, while the relevant band alignment is the least favorable.

OPV is considered to be a rigid system which owes its good conducting properties to the highly conjugated planar structure. Hence, it is somewhat surprising that even when the structure is distorted from planar by bending and twisting the rings, as is the case with the compressed $3.5 \AA$ junction, high conductance is still preserved. An instructive insight into this effect is provided by the comparison of the HOMO of the wires in the equilibrium and compressed junction (shown in Fig. 6). The HOMOs of the free molecule in the equilibrium and distorted geometry, with $\mathrm{H}$ atoms restored, also shown in Fig. 6. indicate that the $\mathrm{H}-\mathrm{S}$ bonds have a similar effect on the frontier orbitals as the Au-S bonds. The qualitatively unchanged shape of the HOMO from the equilibrium planar molecule to the heavily distorted wire in the junction accounts for the nearly constant conductance for a range of contracted gaps. Thus, it is not so much the conformational rigidity of OPV that accounts for the good conducting properties as much as it is the resilience of its $\pi$-conjugated system. Both are provided by the vinylene links, though.

The low bias conductance of tilted OPV3 wires in contracted junctions manifests an opposing trend to that of the compressed distorted wires; namely, it increases with the tilt angle (i.e., gap contraction). This effect has recently been observed experimentally by Haiss et al. for a series of "rigid" molecular wires, ${ }^{12,13}$ including an oligo-phenylene- 


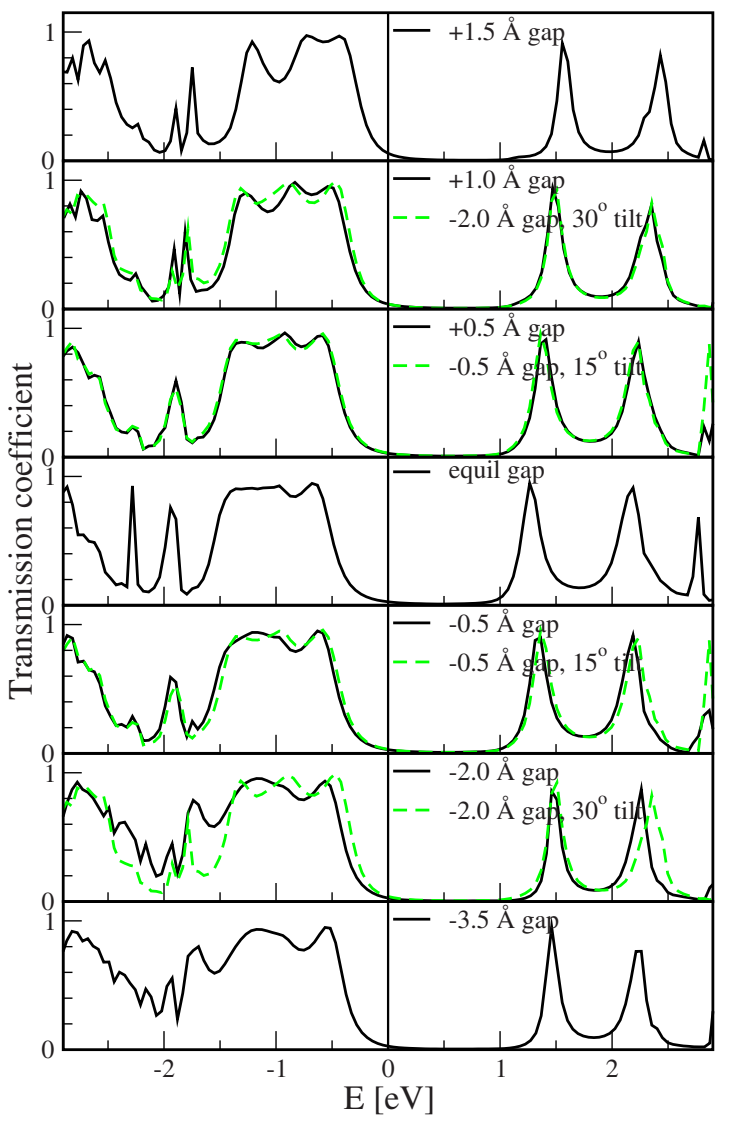

FIG. 5. (Color online) The zero-voltage transmission through the OPV3 wire in extended (three top panels) and compressed (three bottom panels) states. The fourth panel shows the transmission probability at the equilibrium junction geometry. The dashed line shows the transmission coefficient for the the OPV 3 wire in the 0.5 and $2.0 \AA$ contracted junction tilted at an angle of $15^{\circ}$ (panels three and five) and $30^{\circ}$ (panels two and six), respectively. The energy is given relative to the Fermi level, $E_{F}$.

ethynylene derivative with three rings (OPE3), similar to the OPV3. An explanation of this behavior is provided by the zero bias transmission spectra in Fig. 5. The fifth and sixth panels show the spectra for the $15^{\circ}$ and $30^{\circ}$ tilted OPV3 wires in the 0.5 and $2.0 \AA$ contracted junctions, respectively, against their distorted counterparts. It is evident that contribution around the Fermi level increases with the tilt angle, as a consequence of the better band alignment. The distorted wires do not show such a clear trend. In fact the spectra of the distorted and tilted wires do not even look similar. However, if the latter are plotted against those of the elongated junctions, as in the second and third panels of Fig. 5, the resemblance is remarkable. Hence, similar low bias conductances are obtained for the 0.5 and $1.0 \AA$ stretched junctions as for the 0.5 and $2.0 \AA$ contracted junctions, respectively, shown in Table I. While this effect might seem unusual, a closer look into the relevant geometrical parameters of the tilted wires shows it is perfectly plausible. Namely, although the gaps between the leads are contracted, the $\Delta \mathrm{S}-\mathrm{S}$ values in the Table I show that the tilted wires are actually elongated. In the case of the $15^{\circ}$ tilted wire, the elongation is small, only $0.05 \AA$, and thus its spectrum should look more like
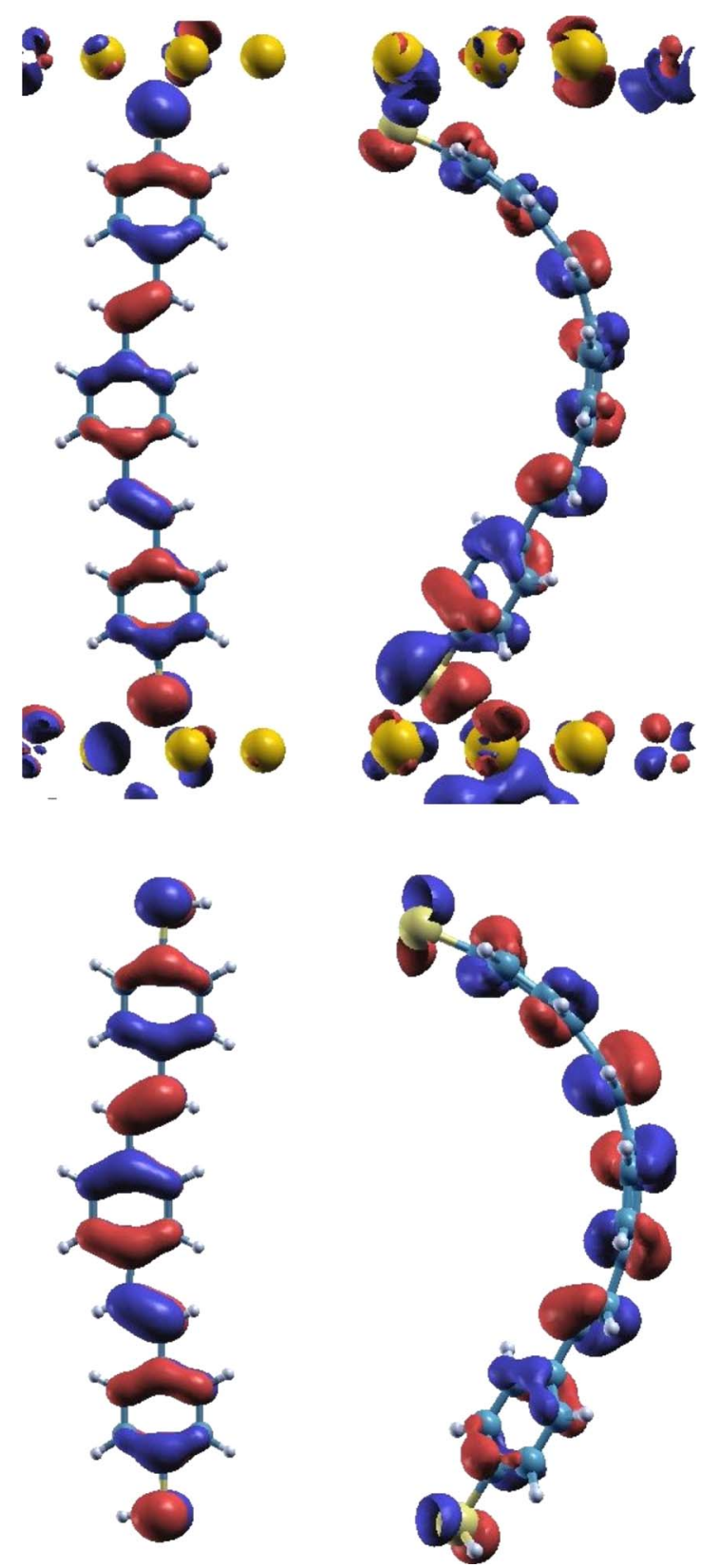

FIG. 6. (Color online) Top: the shape of the HOMO of OPV3 in the equilibrium and $3.5 \AA$ compressed junction. Bottom: the HOMO of the isolated OPV 3 molecule in the same backbone geometry (hydrogen atoms restored). Graphic produced with XCrySDen (Ref. 43) package.

that of the wire at the equilibrium position. Likewise, for the $30^{\circ}$ tilted wire the elongation is slightly below that of the $0.5 \AA$ elongated junction, while its spectrum and the conductance is closer to that of the $1.0 \AA$ case. Clearly, the elongation of the wires is not the reason for their similar conducting properties. Rather, it is the similar nature of the electronic coupling to the electrodes that results in the similar spectra and conductance magnitudes. Because of the unfavorable bond angles between the $\mathrm{C}-\mathrm{S}$ and $\mathrm{S}-\mathrm{Au}$ bonds in the tilted orientation, the binding is weakened, which again yields a better band alignment. Alternatively, one can view the process of tilting the wire as that of keeping the wire 
upright and turning the contacts around sulfur hinges. This puts some strain on the S-Au bonds and, in turn, also on the backbone. But the key effect is the reduced binding at the interface in an approximately symmetric fashion at both ends. Originally the observed behavior was ascribed to an increased coupling strength and broader resonances. ${ }^{12,13}$ The present findings oppose and challenge this view. Provided our interpretations of those measurements are correct, the desirable conductance regime through molecular wires would be achievable simply through an accurate manipulation of contact gap and wire orientation. In view of these findings, our predictions regarding the enhanced conductance of the OPV3 wire with symmetric separation from the contacts appear realistic.

\section{CONCLUSIONS}

The conductance of a single-molecule OPV3 wire between flat gold contacts has been modeled using the NEGF based on DFT calculations. The effects of the contact gap geometry on the conductance have been investigated. It has been predicted that a gap elongation over a small range results in enhanced transport properties of the junction. In con- trast, the contraction of the gap that results in significant molecular distortions is found to have little effect on the charge transport. Finally, the contraction of the gap that causes the wire to tilt at an angle from the surface normal is found to be geometrically analogous to the case of a symmetrically stretched junction, with similarly improved transport properties. The latter findings confirm previous experimental observations, while providing a simple interpretation. Given the "rigid" conjugated frontier orbitals of the wire, all the present results are interpreted in terms of the better band alignment of the nonequilibrium geometries at the expense of coupling strength with the current/voltage electrodes.

\section{ACKNOWLEDGMENTS}

A.B. would like to thank Gemma C. Solomon for critical reading of the manuscript. J.D.G. would like to thank the Australian Research Council for financial support through the Discovery program. The Smeagol program (S.S.) is sponsored by Science Foundation of Ireland. The work in Zagreb was supported by the Croatian Ministry of Science through the Research Projects No. 035-0352828-2839 and No. 0980352828-3118.

*ante.bilic@csiro.au

${ }^{1}$ N. J. Tao, Nat. Nanotechnol. 1, 173 (2006).

${ }^{2}$ J. H. Burroughes, D. D. C. Bradley, A. R. Brown, R. N. Marks, K. Mackay, R. H. Friend, P. L. Burns, and A. B. Holmes, Nature (London) 347, 539 (1990).

${ }^{3}$ S. Kubatkin, A. Danilov, M. Hjort, J. Cornil, J. L. Brédas, N. Stuhr-Hansen, P. Hedegård, and T. Bjørnholm, Nature (London) 425, 698 (2003).

${ }^{4}$ E. A. Osorio, K. O’Neill, M. Wegewijs, N. Stuhr-Hansen, J. Paske, T. Bjørnholm, and H. S. J. van der Zant, Nano Lett. 7, 3336 (2007).

${ }^{5}$ A. V. Danilov, S. Kubatkin, S. Kafanov, K. Flensberg, and T. Bjørnholm, Nano Lett. 8, 2184 (2008).

${ }^{6}$ A. Danilov, S. Kubatkin, S. Kafanov, P. Hedegård, N. StuhrHansen, K. Moth-Poulsen, and T. Bjørnholm, Nano Lett. 8, 1 (2008).

${ }^{7}$ S. M. Lindsay and M. A. Ratner, Adv. Mater. 19, 23 (2007).

${ }^{8}$ C. Toher and S. Sanvito, Phys. Rev. Lett. 99, 056801 (2007).

${ }^{9}$ D. Krüger, H. Fuchs, R. Rousseau, D. Marx, and M. Parrinello, Phys. Rev. Lett. 89, 186402 (2002).

${ }^{10}$ M. G. Roper, M. P. Skegg, C. J. Fisher, J. J. Lee, V. R. Dhanak, D. P. Woodruff, and R. G. Jones, Chem. Phys. Lett. 389, 87 (2004).

${ }^{11}$ K.-H. Müller, Phys. Rev. B 73, 045403 (2006).

${ }^{12}$ W. Haiss, C. Wang, I. Grace, A. S. Batsanov, D. J. Schiffrin, S. J. Higgins, M. R. Bryce, C. J. Lambert, and R. J. Nichols, Nature Mater. 5, 995 (2006).

${ }^{13}$ W. Haiss et al., J. Phys.: Condens. Matter 20, 374119 (2008).

${ }^{14}$ D. Sánchez-Portal, P. Ordejón, E. Artacho, and J. M. Soler, Int. J. Quantum Chem. 65, 453 (1997).

${ }^{15}$ J. M. Soler, E. Artacho, J. D. Gale, A. García, J. Junquera, P. Ordejón, and D. Sánchez-Portal, J. Phys.: Condens. Matter 14,

2745 (2002).

${ }^{16}$ L. Kleinman and D. M. Bylander, Phys. Rev. Lett. 48, 1425 (1982).

${ }^{17}$ N. Troullier and J. L. Martins, Phys. Rev. B 43, 1993 (1991).

${ }^{18}$ J. P. Perdew, K. Burke, and M. Ernzerhof, Phys. Rev. Lett. 77, 3865 (1996).

${ }^{19}$ O. F. Sankey and D. J. Niklewski, Phys. Rev. B 40, 3979 (1989).

${ }^{20}$ J. Junquera, Ó. Paz, D. Sánchez-Portal, and E. Artacho, Phys. Rev. B 64, 235111 (2001).

${ }^{21}$ R. C. Hoft, M. J. Ford, and M. B. Cortie, Mol. Simul. 33, 897 (2007).

${ }^{22}$ M. J. Ford, R. C. Hoft, A. M. McDonagh, and M. B. Cortie, J. Phys.: Condens. Matter 20, 374106 (2008).

${ }^{23}$ P. S. Damle, A. W. Ghosh, and S. Datta, in Molecular Nanoelectronics, edited by M. A. Reed and T. Lee (American Scientific Publishers, Los Angeles, 2003), p. 115

${ }^{24}$ A. R. Rocha, V. M. Garcia-Suarez, S. W. Bailey, C. J. Lambert, J. Ferrer, and S. Sanvito, Nature Mater. 4, 335 (2005).

${ }^{25}$ A. R. Rocha, V. M. Garcia-Suarez, S. W. Bailey, C. J. Lambert, J. Ferrer, and S. Sanvito, Phys. Rev. B 73, 085414 (2006).

${ }^{26}$ I. Rungger and S. Sanvito, Phys. Rev. B 78, 035407 (2008).

${ }^{27}$ A. Bilić, J. R. Reimers, and N. S. Hush, J. Chem. Phys. 122, 094708 (2005).

${ }^{28}$ A. Bilić, J. R. Reimers, N. S. Hush, and J. Hafner, J. Chem. Phys. 116, 8981 (2002).

${ }^{29}$ A. Bilić, J. R. Reimers, and N. S. Hush, J. Phys. Chem. B 106, 6740 (2002).

${ }^{30}$ J. Zhang, A. Bilić, J. R. Reimers, N. S. Hush, and J. Ulstrup, J. Phys. Chem. B 109, 15355 (2005).

${ }^{31}$ A. Bilić, J. R. Reimers, N. S. Hush, R. C. Hoft, and M. J. Ford, J. Chem. Theory Comput. 2, 1093 (2006).

${ }^{32}$ S. Piana and A. Bilic, J. Phys. Chem. B 110, 23467 (2006). 
${ }^{33}$ P. F. Cafe, A. G. Larsen, W. Yang, A. Bilic, I. M. Blake, M. J. Crossley, J. Zhang, H. Wackerbarth, J. Ulstrup, and J. R. Reimers, J. Phys. Chem. C 111, 17285 (2007).

${ }^{34}$ J. R. Reimers, G. C. Solomon, A. Gagliardi, A. Bilić, N. S. Hush, T. Frauenheim, A. D. Carlo, and A. Pecchia, J. Phys. Chem. A 111, 5692 (2007).

${ }^{35}$ M. Hasan, D. Bethell, and M. Brust, J. Am. Chem. Soc. 124, 1132 (2002).

${ }^{36}$ Ž. Crljen, A. Grigoriev, G. Wendin, and K. Stokbro, Phys. Rev. B 71, 165316 (2005).
${ }^{37}$ Y. Xue and M. A. Ratner, Phys. Rev. B 68, 115407 (2003).

${ }^{38}$ V. M. García-Suárez, T. K. S. Bailey, C. Lambert, and B. R. Bulka, Phys. Status Solidi B 244, 2443 (2007).

${ }^{39}$ C. Toher and S. Sanvito, Phys. Rev. B 77, 155402 (2008).

${ }^{40}$ D. Q. Andrews, R. Cohen, R. P. V. Duyne, and M. A. Ratner, J. Chem. Phys. 125, 174718 (2006).

${ }^{41}$ R. C. Hoft, M. J. Ford, V. M. Garcia-Suarez, C. J. Lambert, and M. B. Cortie, J. Phys.: Condens. Matter 20, 025207 (2008).

${ }^{42}$ S. D. Fleming and A. L. Rohl, Z. Kristallogr. 220, 580 (2005).

${ }^{43}$ A. Kokalj, Comput. Mater. Sci. 28, 155 (2003). 